\title{
Observation of the Decay $B_{c}^{+} \rightarrow B_{s}^{0} \pi^{+}$
}

\author{
R. Aaij et al.* \\ (LHCb Collaboration)
}

(Received 22 August 2013; published 1 November 2013)

\begin{abstract}
The result of a search for the decay $B_{c}^{+} \rightarrow B_{s}^{0} \pi^{+}$is presented, using the $B_{s}^{0} \rightarrow D_{s}^{-} \pi^{+}$and $B_{s}^{0} \rightarrow J / \psi \phi$ channels. The analysis is based on a data sample of $p p$ collisions collected with the LHCb detector, corresponding to an integrated luminosity of $1 \mathrm{fb}^{-1}$ taken at a center-of-mass energy of $7 \mathrm{TeV}$, and $2 \mathrm{fb}^{-1}$ taken at $8 \mathrm{TeV}$. The decay $B_{c}^{+} \rightarrow B_{s}^{0} \pi^{+}$is observed with significance in excess of 5 standard deviations independently in both decay channels. The measured product of the ratio of cross sections and branching fraction is $\left[\sigma\left(B_{c}^{+}\right) / \sigma\left(B_{s}^{0}\right)\right] \times \mathcal{B}\left(B_{c}^{+} \rightarrow B_{s}^{0} \pi^{+}\right)=\left[2.37 \pm 0.31\right.$ (stat) \pm 0.11 (syst) $\left.{ }_{-0.13}^{+0.17}\left(\tau_{B_{c}^{+}}\right)\right] \times 10^{-3}$, in the pseudorapidity range $2<\eta(B)<5$, where the first uncertainty is statistical, the second is systematic, and the third is due to the uncertainty on the $B_{c}^{+}$lifetime. This is the first observation of a $B$ meson decaying to another $B$ meson via the weak interaction.
\end{abstract}

DOI: 10.1103/PhysRevLett.111.181801

PACS numbers: $13.25 . \mathrm{Hw}, 12.15 . \mathrm{Ji}$

The $B_{c}^{+}$meson is the ground state of the $\bar{b} c$ system. As such it is unique as it is the only weakly decaying doubly heavy meson. All measurements of $B_{c}^{+}$meson decays to date are decays where the constituent $b$ quark decays weakly to a $c$ quark [1-8]. The decay of the $B_{c}^{+}$meson to another $B$ meson, with the bottom quark acting as a spectator (see Fig. 1), has not previously been observed. This will improve the understanding of theoretical predictions and provide valuable information for the source of $B_{s}^{0}$ mesons at the LHC.

A wide range of predictions for the branching fraction $\mathcal{B}\left(B_{c}^{+} \rightarrow B_{s}^{0} \pi^{+}\right)$exists, between $16.4 \%$ and $2.5 \%$, based on, e.g., QCD sum rules $[9,10]$, or quark-potential models (see Refs. [11-16] and references therein). Experimental clarification is needed to shed light on the present theoretical status. Unlike most other $B$ decays, the higher order corrections in the expansion of Heavy Quark Effective Theory within the framework of quantum chromodynamics (QCD) are relatively large. The expansion is described in powers of $m_{c} / m_{b}$ rather than $\Lambda_{\mathrm{QCD}} / m_{b}$, due to the presence of two heavy quark constituents, where $\Lambda_{\mathrm{QCD}}$ is the QCD scale, and $m_{c}\left(m_{b}\right)$ the charm (bottom) quark mass. In addition, the energy release in the decay is relatively small, leading to larger nonfactorizable effects compared to decays with lighter daughter particles. Study of the decay $B_{c}^{+} \rightarrow B_{s}^{0} \pi^{+}$allows these models to be tested. Knowledge of the production of $B_{s}^{0}$ mesons from $B_{c}^{+}$ decays is also useful for time-dependent analyses of $B_{s}^{0}$ decays, to understand any associated decay-time bias due to the incorrect estimate of the $B_{s}^{0}$ decay time if

*Full author list given at end of the article.

Published by the American Physical Society under the terms of the Creative Commons Attribution 3.0 License. Further distribution of this work must maintain attribution to the author(s) and the published article's title, journal citation, and DOI. originating from a $B_{c}^{+}$decay, or to take advantage of flavor tagging capabilities using the accompanying ("bachelor") pion.

The data used in this analysis were collected with the LHCb detector [17] from $p p$ collisions at $\sqrt{s}=7 \mathrm{TeV}$ and $8 \mathrm{TeV}$, corresponding to integrated luminosities of $1 \mathrm{fb}^{-1}$ and $2 \mathrm{fb}^{-1}$, respectively.

The decays $B_{s}^{0} \rightarrow D_{s}^{-} \pi^{+}$and $B_{s}^{0} \rightarrow J / \psi \phi$ are used, with the subsequent decays $D_{s}^{-} \rightarrow K^{+} K^{-} \pi^{-}, J / \psi \rightarrow$ $\mu^{+} \mu^{-}$and $\phi \rightarrow K^{+} K^{-}$. The inclusion of charge conjugate modes is implied throughout. The event selection and fits to the $B_{s}^{0}$ invariant mass distributions follow previous LHCb analyses based on these $B_{s}^{0}$ decay modes $[18,19]$. The two channels are analyzed independently and the final results are combined. The strategy is to normalize the final number of $B_{c}^{+} \rightarrow B_{s}^{0} \pi^{+}$decays to the number of $B_{s}^{0}$ decays, which gives a result for the $B_{c}^{+} \rightarrow B_{s}^{0} \pi^{+}$branching fraction multiplied by the ratio of $B_{c}^{+}$and $B_{s}^{0}$ production rates, $\left[\sigma\left(B_{c}^{+}\right) / \sigma\left(B_{s}^{0}\right)\right] \times \mathcal{B}\left(B_{c}^{+} \rightarrow B_{s}^{0} \pi^{+}\right)$. The $B_{c}^{+}$signal

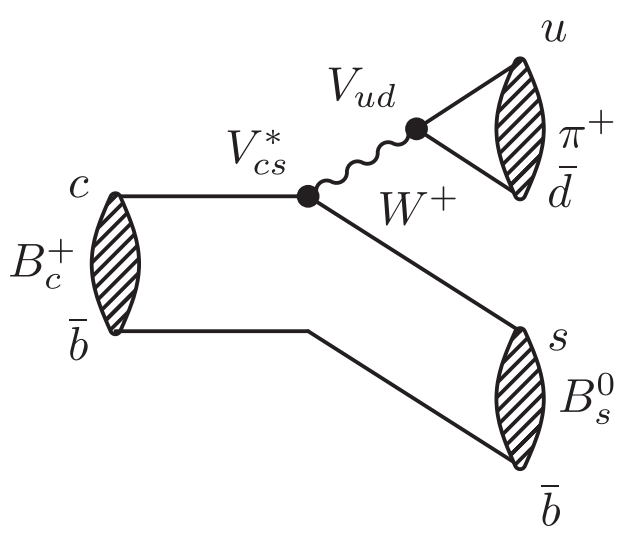

FIG. 1. Leading-order Feynman diagram of the decay $B_{c}^{+} \rightarrow B_{s}^{0} \pi^{+}$. 
region was not examined until the event selection was finalized. Since the ratio of production rates, $\sigma\left(B_{c}^{+}\right) / \sigma\left(B_{s}^{0}\right)$, may depend on the kinematics of the produced $B$ meson, the result is quoted for $B$ mesons produced in the pseudorapidity range $2<\eta(B)<5$, corresponding to the $\mathrm{LHCb}$ detector acceptance.

The LHCb detector is a single-arm forward spectrometer covering the pseudorapidity range $2<\eta<5$, described in detail in Ref. [17]. The combined tracking system provides momentum measurement with relative uncertainty that varies from $0.4 \%$ at $5 \mathrm{GeV} / c$ to $0.6 \%$ at $100 \mathrm{GeV} / c$, and impact parameter resolution of $20 \mu \mathrm{m}$ for tracks with high transverse momentum, $p_{T}$. The impact parameter (IP) is defined as the distance of closest approach between the track and a primary interaction. Charged hadrons are identified using two ring-imaging Cherenkov detectors. The charged pions from $B_{c}^{+}$decays are selected with an efficiency of $93 \%$ while keeping the misidentification rate of kaons below 7\%. Muons are identified by a system composed of alternating layers of iron and multiwire proportional chambers with a typical efficiency of $97 \%$ at $1 \%-3 \%$ pion to muon misidentification probability. The trigger [20] consists of a hardware stage, based on information from the calorimeter and muon systems, followed by a software stage, which applies a full event reconstruction. The $B_{s}^{0}$ candidates with muons in the final state are required to pass the hardware trigger, which selects muons with a transverse momentum, $p_{T}>1.48 \mathrm{GeV} / c$, whereas the $B_{s}^{0}$ candidates with only hadrons in the final state are selected by requiring a hadron in the calorimeter with $E_{T}>3.6 \mathrm{GeV} / c$.

Monte Carlo simulations, used to develop the $B_{c}^{+}$candidate selection, are performed using BCVEGPY [21], interfaced with PYTHIA 6.4 [22] using a specific LHCb configuration [23]. Decays of hadronic particles are described by EVTGEN [24], in which final state radiation is generated using PHOTOS [25]. The interaction of the generated particles with the detector and its response are implemented using the GEANT4 toolkit [26] as described in Ref. [27].
The $B_{s}^{0}$ candidates are selected using the multivariate analysis known as the boosted decision tree (BDT) $[28,29]$, to optimally discriminate between signal and background. In the training, simulated $B_{s}^{0}$ decays are used as the signal, whereas candidates in the $B_{s}^{0}$ mass sideband in data are used as the background. To avoid potential biases, only one sixth of the data is used in the training. It is verified that the distribution of the BDT discriminant is the same for the events used in the training, compared to those that were not. All events are used for the final result. The BDT training for the selection of $B_{s}^{0} \rightarrow D_{s}^{-} \pi^{+}$candidates uses only the upper sideband $[5466,5800] \mathrm{MeV} / c^{2}$, as the lower sideband contains a large amount of irreducible partially reconstructed $B$ decays, while the training for $B_{s}^{0} \rightarrow J / \psi \phi$ uses both lower sideband [5200, 5316] MeV/ $/ c^{2}$ and upper mass sideband $[5416,5550] \mathrm{MeV} / c^{2}$. The $B_{s}^{0}$ vertex quality $\left(\chi_{\mathrm{vtx}}^{2}\right)$, flight distance, momentum $p$, and $p_{T}$ are used to discriminate the signal from the background. For the $D_{s}^{-} \pi^{+}$final state we use, in addition, the $\chi_{\mathrm{vtx}}^{2}$, flight distance, $p$ and $p_{T}$ of the $D_{s}^{-}$candidate and the $p, p_{T}$ and $\chi_{\mathrm{IP}}^{2}$ of the bachelor pion from the $B_{s}^{0}$ decay to suppress combinatorial background. The quantity $\chi_{\mathrm{IP}}^{2}$ is defined as the difference in $\chi^{2}$ of a given primary vertex $(\mathrm{PV})$ reconstructed with and without the considered track. The training for $J / \psi \phi$ candidates uses $p, p_{T}, \chi_{\mathrm{vtx}}^{2}$, and $\chi_{\mathrm{IP}}^{2}$ of the $J / \psi$ and $\phi$ candidates, and the $p_{T}$ of the final state kaons and muons. In the selection of $B_{s}^{0}$ candidates from $B_{c}^{+}$ decays, variables that require the candidate to point to a primary vertex, such as the impact parameter of the $B_{s}^{0}$ candidate, are explicitly not included. The minimum value of the BDT discriminant is chosen by optimizing the $B_{s}^{0}$ signal significance $S / \sqrt{S+B}$, where $S$ and $B$ are the expected numbers of signal and combinatorial background events, respectively.

The total number of $B_{s}^{0}$ decays is obtained from extended unbinned maximum likelihood fits to the invariant mass distributions, using mass constraints for the $J / \psi$ candidates [30], and are shown in Fig. 2. The signal shapes are taken as double Crystal Ball functions [31] with a common peak value and with tails to either side of the
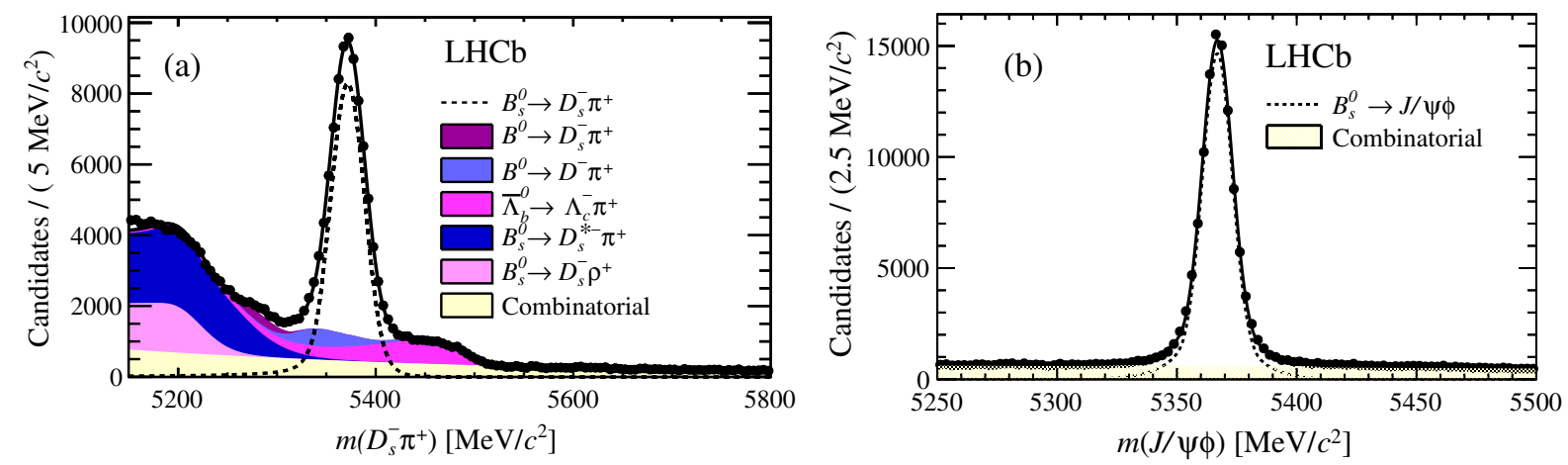

FIG. 2 (color online). Invariant mass distributions of (a) $B_{s}^{0} \rightarrow D_{s}^{-} \pi^{+}$and (b) $B_{s}^{0} \rightarrow J / \psi \phi$ candidates. The different components are defined in the legend. 
peak, to account for final state radiation and detector resolution effects. The parameters that describe the tails are obtained from simulation and are fixed in the fits. The peak and width parameters of the signal are allowed to vary. The combinatorial backgrounds are modeled with exponential distributions. The $B_{s}^{0} \rightarrow D_{s}^{-} \pi^{+}$final state is contaminated by partially reconstructed $B$ decays such as $B_{s}^{0} \rightarrow D_{s}^{*-} \pi^{+}$and $B_{s}^{0} \rightarrow D_{s}^{-} \rho^{+}$decays, where the soft photon or neutral pion is not reconstructed, and by decays where one of the final state particles is misidentified as a kaon, such as $B^{0} \rightarrow D^{-} \pi^{+}$or $\bar{\Lambda}_{b}^{0} \rightarrow \Lambda_{c}^{-} \pi^{+}$decays. The shapes of these backgrounds are fixed from simulation, following Ref. [18]. In total $103760 \pm 380 B_{s}^{0} \rightarrow J / \psi \phi$ and $73700 \pm 500 B_{s}^{0} \rightarrow D_{s}^{-} \pi^{+}$decays are found.

Selected $B_{s}^{0}$ candidates with masses consistent with the known $B_{s}^{0}$ mass are combined with tracks that satisfy loose pion identification requirements. Subsequently, $B_{c}^{+}$candidates are selected with a second BDT algorithm. In the training of the second BDT, simulated candidates with masses consistent with the $B_{c}^{+}$mass [32] are used as the signal, and candidates in the $B_{c}^{+}$mass sideband region in data are used as the background. For this, only the upper mass sideband is used in the case of $B_{s}^{0} \rightarrow D_{s}^{-} \pi^{+}$, while also the lower mass sideband is used in the case of $B_{s}^{0} \rightarrow$ $J / \psi \phi$, to further suppress the larger combinatorial background at smaller values of the mass. Only one sixth of the total data set is used in the training. The second BDT uses the following variables: the $B_{c}^{+}$candidate $p_{T}$, decay time, $\chi_{\mathrm{vtx}}^{2}, \chi_{\mathrm{IP}}^{2}$, and the $B_{c}^{+}$pointing angle, i.e., the angle between the $B_{c}^{+}$candidate momentum vector and the line joining the associated $\mathrm{PV}$ and the $B_{c}^{+}$decay vertex. The $B_{s}^{0}$ polar angle (the angle between $B_{s}^{0}$ flight direction and the beam axis), decay time, decay length, and pointing angle are also used. The $p$ and $p_{T}$ of the bachelor pion from the $B_{c}^{+}$decay are the most discriminating observables in the second BDT. Differences between the analyses of the $D_{s}^{-} \pi^{+}$and $J / \psi \phi$ final states are the use of $\chi_{\mathrm{IP}}^{2}$ of the $B_{s}^{0}$ candidate and bachelor pion (from the $B_{c}^{+}$decay), and $B_{s}^{0}$ and $B_{c}^{+}$momentum for the former, and the use of the $B_{c}^{+}$and $B_{s}^{0}$ decay-length uncertainties for the latter. The optimal selections are defined by maximizing figures of merit for a target level of significance of 3 standard deviations, $\epsilon /(3 / 2+\sqrt{B})$ [33], where $\epsilon$ is the signal efficiency for a given BDT criterion. The figure of merit displays a plateau, and the chosen value is at the lower end to allow us to better constrain the shape of the combinatorial background. The chosen selection is very close to the optimal point for a target level of $5 \sigma$ and for the expected significance $S / \sqrt{S+B}$. The trigger for $B_{s}^{0} \rightarrow D_{s}^{-} \pi^{+}$decays preferentially selects candidates with high $p_{T}$ with respect to the trigger for $B_{s}^{0} \rightarrow J / \psi \phi$ decays, which results in higher efficiency for the second BDT requirement for the $B_{s}^{0} \rightarrow D_{s}^{-} \pi^{+}$final state. The $B_{c}^{+}$and $B_{s}^{0}$ candidates are required to be produced in the pseudorapidity range $2<\eta(B)<5$.

The invariant mass distributions for the $B_{c}^{+} \rightarrow B_{s}^{0} \pi^{+}$ candidates are shown in Fig. 3, together with the resulting fits. The decay $B_{c}^{+} \rightarrow B_{s}^{0} \pi^{+}$has a $Q$ value of $770 \mathrm{MeV} / c^{2}$ (with $Q \equiv m_{B_{c}^{+}}-m_{B_{s}^{0}}-m_{\pi^{+}}$), which results in a resolution of about $6 \mathrm{MeV} / c^{2}$ when a $B_{s}^{0}$ mass constraint is applied. The signal shape is modeled as a double Crystal Ball function, with its parameters obtained from simulated events. The larger number of $B_{c}^{+}$candidates in the $B_{s}^{0} \rightarrow$ $D_{s}^{-} \pi^{+}$channel allows variation of the peak position and the width in the fit. The combinatorial background is primarily due to signal $B_{s}^{0}$ decays combined with a random pion from the primary vertex, and is modeled with an exponential function. Backgrounds due to $B_{c}^{+} \rightarrow B_{s}^{*} \pi^{+}$ and $B_{c}^{+} \rightarrow B_{s}^{0} \rho^{+}$decays, where the photon or neutral pion are not reconstructed, are simulated, and their shapes are modeled with Gaussian distributions, with parameters fixed in the fit, and yields allowed to vary. Statistical signal significances of $7.7 \sigma$ for $B_{c}^{+} \rightarrow B_{s}^{0}\left(\rightarrow D_{s}^{-} \pi^{+}\right) \pi^{+}$and $6.1 \sigma$ for $B_{c}^{+} \rightarrow B_{s}^{0}(\rightarrow J / \psi \phi) \pi^{+}$decays are obtained from the likelihood ratio of fits with and without the probability density function for the signal shape, $\sqrt{-2 \ln \left(\mathcal{L}_{B} / \mathcal{L}_{S+B}\right)}$, with $64 \pm 10$ and $35 \pm 8$ signal decays, respectively.
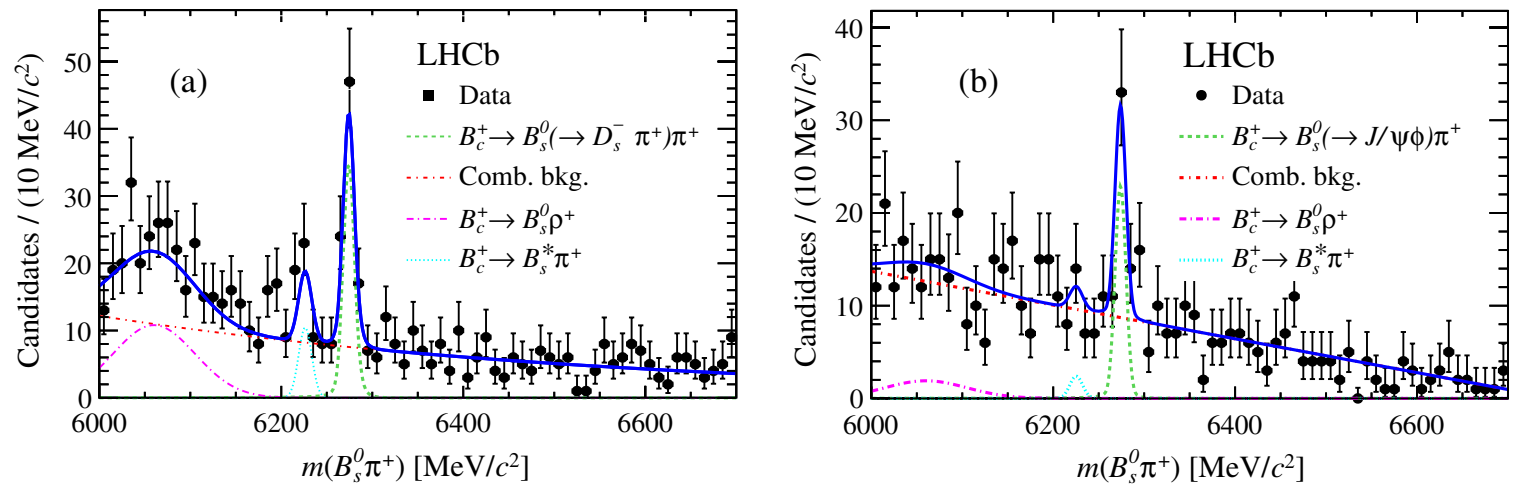

FIG. 3 (color online). $B_{c}^{+}$mass fits for the combined 2011 and 2012 data sets for (a) $B_{c}^{+} \rightarrow B_{s}^{0}\left(\rightarrow D_{s}^{-} \pi^{+}\right) \pi^{+}$and (b) $B_{c}^{+} \rightarrow B_{s}^{0}(\rightarrow J / \psi \phi) \pi^{+}$candidates. The different components are indicated in the legends. 
In Fig. 3(a), the structure around $6225 \mathrm{MeV} / c^{2}$ is consistent with originating from $B_{c}^{+} \rightarrow B_{s}^{*} \pi^{+}$decays. However, this contribution is not significant.

To obtain the value for the $B_{c}^{+} \rightarrow B_{s}^{0} \pi^{+}$branching fraction, multiplied by the ratio of $B_{c}^{+}$and $B_{s}^{0}$ production rates, the relative detection efficiency of $B_{s}^{0}$ decays compared to $B_{c}^{+} \rightarrow B_{s}^{0} \pi^{+}$decays is determined from simulation. Requiring the bachelor pion to be inside the LHCb acceptance reduces the $B_{c}^{+} \rightarrow B_{s}^{0} \pi^{+}$yield by about $19 \%$ with respect to the $B_{s}^{0}$ yield. The most significant reduction in the number of selected $B_{c}^{+}$candidates comes from suppressing $B_{s}^{0}$ combinations with a random pion from the primary interaction, by means of the second BDT selection. The total relative detection efficiency of $B_{c}^{+} \rightarrow B_{s}^{0} \pi^{+}$ decays with respect to $B_{s}^{0}$ decays is estimated to be $15.2 \%$ for the $B_{s}^{0} \rightarrow J / \psi \phi$ decay and $33.9 \%$ for the $B_{s}^{0} \rightarrow D_{s}^{-} \pi^{+}$ final state. This difference in $B_{c}^{+}$selection efficiencies is a consequence of the difference in $B_{s}^{0}$ trigger and selection requirements.

The sources of systematic uncertainty for the efficiencycorrected ratio of $B_{c}^{+}$and $B_{s}^{0}$ yields are listed in Table I. The uncertainty on the $B_{s}^{0}$ yield in the $D_{s}^{-} \pi^{+}$analysis is determined by varying the parameters that describe the tails of the signal mass distribution, and by reducing the exponent of the combinatorial background by a factor of 2. The uncertainty on the $B_{s}^{0} \rightarrow J / \psi \phi$ yield is obtained by comparing the fitted yield in simulated pseudoexperiments to the yield that was used as input to those experiments.

The uncertainty on the $B_{c}^{+}$yield is quantified by varying the peak position and width in the fit to $B_{c}^{+} \rightarrow$ $B_{s}^{0}(\rightarrow J / \psi \phi) \pi^{+}$candidates. The signal model is validated using simulated pseudoexperiments in the $J / \psi \phi$ analysis, whereas the tail parameters are varied by $\pm 10 \%$ in the $D_{s}^{-} \pi^{+}$analysis. In addition, the combinatorial background

TABLE I. Contributions of the various sources of (relative) systematic uncertainty on the efficiency-corrected ratio of event yields. The total systematic uncertainty is the quadratic sum of the individual contributions. The number of $B_{c}^{+} \rightarrow B_{s}^{0}\left(\rightarrow D_{s}^{-} \pi^{+}\right) \pi^{+}$candidates is large enough that the peak position and width are freely varied in the fit, and hence the corresponding uncertainty is contained in the statistical uncertainty of the signal yield.

\begin{tabular}{lcc}
\hline \hline Source & $D_{s}^{-} \pi^{+}(\%)$ & $J / \psi \phi(\%)$ \\
\hline$B_{s}^{0}$ fit model & 3.0 & 1.2 \\
$B_{c}^{+}$mean mass & $\ldots$ & 2.0 \\
$B_{c}^{+}$mass resolution & $\ldots$ & 5.2 \\
$B_{c}^{+}$signal model & 1.5 & 1.7 \\
Combinatorial background model & 1.8 & 0.3 \\
Partially reconstructed background & 1.8 & 1.7 \\
Data-simulation difference & 3.7 & 3.7 \\
$B_{c}^{+}$lifetime & +6.8 & 7.4 \\
Total & -3.5 & 10.4 \\
\hline \hline
\end{tabular}

shape is changed to a straight line, and the difference in the signal yield is taken as the associated systematic uncertainty. The effect of partially reconstructed $B_{c}^{+} \rightarrow B_{s}^{0} \rho^{+}$ decays is estimated by excluding candidates with mass less than $6150 \mathrm{MeV} / c^{2}$ from the fit. The significance of the $B_{c}^{+} \rightarrow B_{s}^{0} \pi^{+}$signal is reduced to $7.5 \sigma$ for $B_{c}^{+} \rightarrow$ $B_{s}^{0}\left(\rightarrow D_{s}^{-} \pi^{+}\right) \pi^{+}$and $5.5 \sigma$ for $B_{c}^{+} \rightarrow B_{s}^{0}(\rightarrow J / \psi \phi) \pi^{+}$ when the systematic uncertainties on the fit to the $B_{c}^{+}$ mass distribution are taken into account.

The relative detection efficiency of $B_{c}^{+}$and $B_{s}^{0}$ events is determined from simulated events. The correspondence between data and simulation is quantified by varying the criterion on the BDT value, and by comparing the observed $B_{s}^{0}$ yield to the expected yield based on the change in efficiency as determined from simulation. The largest contribution is due to the $10 \%$ uncertainty on the $B_{c}^{+}$lifetime [32], which was recently improved by the CDF Collaboration [34]. The change in selection efficiency when varying the $B_{c}^{+}$lifetime by $\pm 10 \%$ is assigned as the systematic uncertainty. A longer (shorter) $B_{c}^{+}$lifetime corresponds to a larger (smaller) efficiency and therefore a smaller (larger) ratio. As a cross-check, the effect of the choice of different sets of BDT input variables is investigated and the result is found to be stable.

The contribution from Cabibbo suppressed $B_{c}^{+} \rightarrow B_{s}^{0} K^{+}$ decays, the uncertainty on the efficiency of reconstructing the extra pion, and the uncertainty on the efficiency of the particle identification requirement on the bachelor pion all give small contributions $(<1.0 \%)$ to the total systematic uncertainty, and are not itemized in the summary in Table I.

The $B_{s}^{0}$ and $B_{c}^{+}$yields are corrected for the relative detection efficiencies, to obtain the efficiency-corrected ratios of $B_{c}^{+} \rightarrow B_{s}^{0} \pi^{+}$over $B_{s}^{0}$ yields, $\left[2.54 \pm 0.40\right.$ (stat) ${ }_{-0.17}^{+0.23}($ syst $\left.)\right] \times$ $10^{-3}$ and $[2.20 \pm 0.49$ (stat) \pm 0.23 (syst) $] \times 10^{-3}$ for the $D_{s}^{-} \pi^{+}$and $J / \psi \phi$ final states, respectively. The small fraction of $B_{s}^{0}$ candidates originating from $B_{c}^{+}$decays is neglected. The uncertainty due to the uncertainty on the $B_{c}^{+}$ lifetime is correlated between the two measurements, and is accounted for in the combined result of the ratio of production rates multiplied with the branching fraction

$$
\begin{aligned}
& \frac{\sigma\left(B_{c}^{+}\right)}{\sigma\left(B_{s}^{0}\right)} \times \mathcal{B}\left(B_{c}^{+} \rightarrow B_{s}^{0} \pi^{+}\right) \\
& \quad=\left[2.37 \pm 0.31(\text { stat }) \pm 0.11(\text { syst })_{-0.13}^{+0.17}\left(\tau_{B_{c}^{+}}\right)\right] \times 10^{-3},
\end{aligned}
$$

where the first uncertainty is statistical, the second is systematic, and the third is due to the uncertainty on the $B_{c}^{+}$lifetime. Since $\sigma\left(B_{c}^{+}\right) / \sigma\left(B_{s}^{0}\right)$ may depend on the kinematics of the produced $B$ meson, the data are divided according to center-of-mass energy leading to [1.27 \pm 0.42 (stat) \pm 0.05 (syst $\left.)_{-0.07}^{+0.09}\left(\tau_{B_{c}^{+}}\right)\right] \times 10^{-3}$ and $[2.92 \pm$ 0.40 (stat) \pm 0.12 (syst) $\left.{ }_{-0.16}^{+0.21}\left(\tau_{B_{c}^{+}}\right)\right] \times 10^{-3}$ for $\sqrt{s}=7$ and $8 \mathrm{TeV} p p$ collisions, respectively. The lower value for the 
result of the $7 \mathrm{TeV}$ data is attributed to a downward statistical fluctuation of the $B_{c}^{+} \rightarrow B_{s}^{0}(\rightarrow J / \psi \phi) \pi^{+}$yield in the 2011 data set, with a $p$ value of $1.5 \%$.

Assuming a value for $\mathcal{B}\left(B_{c}^{+} \rightarrow J / \psi \pi^{+}\right)$around $0.15 \%$ [11], combined with the results $\left[\sigma\left(B_{c}^{+}\right) / \sigma\left(B^{+}\right)\right] \times$ $\mathcal{B}\left(B_{c}^{+} \rightarrow J / \psi \pi^{+}\right) / \mathcal{B}\left(B^{+} \rightarrow J / \psi K^{+}\right)=(0.68 \pm 0.10 \pm$ $0.03 \pm 0.05) \%$ [4], and measurements of $f_{s} / f_{d}$ [18] and $\mathcal{B}\left(B^{+} \rightarrow J / \psi K^{+}\right)$[32], results in a ratio of production rates of $B_{c}^{+}$mesons over $B_{s}^{0}$ mesons of about 0.02 . This leads to a branching fraction for $B_{c}^{+} \rightarrow B_{s}^{0} \pi^{+}$of about $10 \%$. Although precise quantification requires improved understanding of $\sigma\left(B_{c}^{+}\right)$and $\mathcal{B}\left(B_{c}^{+} \rightarrow J / \psi \pi^{+}\right)$, even taking the lower estimates for $\mathcal{B}\left(B_{c}^{+} \rightarrow J / \psi \pi^{+}\right)$that are found in the literature [11], leads to a value of $\mathcal{B}\left(B_{c}^{+} \rightarrow B_{s}^{0} \pi^{+}\right)$which is the largest exclusive branching fraction of any known weak $B$ meson decay.

In summary, the first observation of a weak decay of a $B$ meson to another $B$ meson is reported. This measurement will help to better understand flavor tagging and the decay time resolution in time-dependent $B_{s}^{0}$ analyses, and in addition will constrain models that predict branching fractions of $B_{c}^{+}$decays.

We wish to thank A. K. Likhoded for useful discussions. We express our gratitude to our colleagues in the CERN accelerator departments for the excellent performance of the LHC. We thank the technical and administrative staff at the LHCb institutes. We acknowledge support from CERN and from the national agencies: CAPES, CNPq, FAPERJ and FINEP (Brazil); NSFC (China); CNRS/IN2P3 and Region Auvergne (France); BMBF, DFG, HGF and MPG (Germany); SFI (Ireland); INFN (Italy); FOM and NWO (The Netherlands); SCSR (Poland); MEN/IFA (Romania); MinES, Rosatom, RFBR and NRC "Kurchatov Institute" (Russia); MinECo, XuntaGal and GENCAT (Spain); SNSF and SER (Switzerland); NAS Ukraine (Ukraine); STFC (United Kingdom); NSF (USA). We also acknowledge the support received from the ERC under FP7. The Tier1 computing centers are supported by IN2P3 (France), KIT and BMBF (Germany), INFN (Italy), NWO and SURF (Netherlands), PIC (Spain), GridPP (United Kingdom). We are thankful for the computing resources put at our disposal by Yandex LLC (Russia), as well as to the communities behind the multiple open source software packages that we depend on.

[1] F. Abe et al. (CDF Collaboration), Phys. Rev. Lett. 81, 2432 (1998); F. Abe et al. (CDF Collaboration), Phys. Rev. D 58, 112004 (1998).

[2] T. Aaltonen et al. (CDF Collaboration), Phys. Rev. Lett. 100, 182002 (2008).

[3] V. M. Abazov et al. (D0 Collaboration), Phys. Rev. Lett. 101, 012001 (2008).

[4] R. Aaij et al. (LHCb Collaboration), Phys. Rev. Lett. 109, 232001 (2012).
[5] R. Aaij et al. (LHCb Collaboration), Phys. Rev. Lett. 108, 251802 (2012).

[6] R. Aaij et al. (LHCb Collaboration), Phys. Rev. D 87, 071103(R) (2013).

[7] R. Aaij et al. (LHCb Collaboration), Phys. Rev. D 87, 112012 (2013).

[8] R. Aaij et al. (LHCb Collaboration), J. High Energy Phys. 09 (2013) 075.

[9] V. Kiselev, A. Kovalsky, and A. Likhoded, Nucl. Phys. B585, 353 (2000).

[10] I. P. Gouz, V. V. Kiselev, A. K. Likhoded, V. I. Romanovsky, and O.P. Yushchenko, Phys. At. Nucl. 67, 1559 (2004).

[11] M. A. Ivanov, J. G. Korner, and P. Santorelli, Phys. Rev. D 73, 054024 (2006).

[12] A. Y. Anisimov, I. Narodetsky, C. Semay, and B. SilvestreBrac, Phys. Lett. B 452, 129 (1999).

[13] P. Colangelo and F. De Fazio, Phys. Rev. D 61, 034012 (2000).

[14] D. Ebert, R. Faustov, and V. Galkin, Eur. Phys. J. C 32, 29 (2003).

[15] R. Dhir, N. Sharma, and R. Verma, J. Phys. G 35, 085002 (2008).

[16] Sk. Naimuddin, S. Kar, M. Priyadarsini, N. Barik, and P. C. Dash, Phys. Rev. D 86, 094028 (2012).

[17] A. A. Alves, Jr. et al. (LHCb Collaboration), JINST 3, S08005 (2008).

[18] R. Aaij et al. (LHCb Collaboration), J. High Energy Phys. 04 (2013) 001.

[19] R. Aaij et al. (LHCb Collaboration), Phys. Rev. D 87, 112010 (2013).

[20] R. Aaij et al., JINST 8, P04022 (2013).

[21] C.-H. Chang, J.-X. Wang, and X.-G. Wu, Comput. Phys. Commun. 174, 241 (2006).

[22] T. Sjöstrand, S. Mrenna, and P. Skands, J. High Energy Phys. 05 (2006) 026.

[23] I. Belyaev et al., in Handling of the Generation of Primary Events in GAUSS, the LHCb Simulation Framework, Nuclear Science Symposium Conference Record (NSS/ MIC) (IEEE, New York, 2010), p. 1155.

[24] D. J. Lange, Nucl. Instrum. Methods Phys. Res., Sect. A 462, 152 (2001).

[25] P. Golonka and Z. Was, Eur. Phys. J. C 45, 97 (2006).

[26] J. Allison et al. (GEANT4 Collaboration) IEEE Trans. Nucl. Sci. 53, 270 (2006); S. Agostinelli et al. (GEANT4 Collaboration), Nucl. Instrum. Methods Phys. Res., Sect. A 506, 250 (2003).

[27] M. Clemencic, G. Corti, S. Easo, C. R. Jones, S. Miglioranzi, M. Pappagallo, and P. Robbe, J. Phys. Conf. Ser. 331, 032023 (2011).

[28] L. Breiman, J.H. Friedman, R. A. Olshen, and C.J. Stone, Classification and Regression Trees (Wadsworth International Group, Belmont, California, USA, 1984).

[29] R. E. Schapire and Y. Freund, J. Comput. Syst. Sci. 55, 119 (1997).

[30] W. D. Hulsbergen, Nucl. Instrum. Methods Phys. Res., Sect. A 552, 566 (2005).

[31] T. Skwarnicki, Ph.D. thesis, Institute of Nuclear Physics, Krakow, 1986, DESY-F31-86-02. 
[32] J. Beringer et al. (Particle Data Group), Phys. Rev. D 86, 010001 (2012).

[33] G. Punzi, in Sensitivity of Searches for New Signals and its Optimization, edited by L. Lyons, R. Mount, and R. Reitmeyer, Statistical Problems in Particle Physics,
Astrophysics, and Cosmology, econf C030908, SLAC-R703 (2003), p. 79.

[34] T. Aaltonen et al. (CDF Collaboration), Phys. Rev. D 87, 011101 (2013).

R. Aaij, ${ }^{40}$ B. Adeva, ${ }^{36}$ M. Adinolfi, ${ }^{45}$ C. Adrover, ${ }^{6}$ A. Affolder,${ }^{51}$ Z. Ajaltouni, ${ }^{5}$ J. Albrecht,,${ }^{9}$ F. Alessio, ${ }^{37}$ M. Alexander ${ }^{50}$ S. Ali, ${ }^{40}$ G. Alkhazov, ${ }^{29}$ P. Alvarez Cartelle, ${ }^{36}$ A. A. Alves, Jr.,${ }^{24,37}$ S. Amato, ${ }^{2}$ S. Amerio, ${ }^{21}$ Y. Amhis, ${ }^{7}$ L. Anderlini, ${ }^{17, f}$ J. Anderson,${ }^{39}$ R. Andreassen, ${ }^{56}$ J. E. Andrews, ${ }^{57}$ R. B. Appleby,${ }^{53}$ O. Aquines Gutierrez, ${ }^{10}$ F. Archilli, ${ }^{18}$ A. Artamonov, ${ }^{34}$ M. Artuso,${ }^{58}$ E. Aslanides, ${ }^{6}$ G. Auriemma, ${ }^{24, m}$ M. Baalouch, ${ }^{5}$ S. Bachmann, ${ }^{11}$ J. J. Back, ${ }^{47}$ C. Baesso, ${ }^{59}$ V. Balagura,${ }^{30}$ W. Baldini, ${ }^{16}$ R. J. Barlow, ${ }^{53}$ C. Barschel,${ }^{37}$ S. Barsuk, ${ }^{7}$ W. Barter, ${ }^{46}$ Th. Bauer, ${ }^{40}$ A. Bay,${ }^{38}$ J. Beddow,${ }^{50}$ F. Bedeschi,${ }^{22}$ I. Bediaga, ${ }^{1}$ S. Belogurov,${ }^{30}$ K. Belous, ${ }^{34}$ I. Belyaev, ${ }^{30}$

E. Ben-Haim, ${ }^{8}$ G. Bencivenni, ${ }^{18}$ S. Benson, ${ }^{49}$ J. Benton, ${ }^{45}$ A. Berezhnoy, ${ }^{31}$ R. Bernet, ${ }^{39}$ M.-O. Bettler ${ }^{46}$ M. van Beuzekom, ${ }^{40}$ A. Bien, ${ }^{11}$ S. Bifani, ${ }^{44}$ T. Bird,${ }^{53}$ A. Bizzeti, ${ }^{17, h}$ P. M. Bjørnstad,${ }^{53}$ T. Blake, ${ }^{37}$ F. Blanc, ${ }^{38}$

J. Blouw, ${ }^{10}$ S. Blusk,${ }^{58}$ V. Bocci, ${ }^{24}$ A. Bondar,${ }^{33}$ N. Bondar, ${ }^{29}$ W. Bonivento, ${ }^{15}$ S. Borghi, ${ }^{53}$ A. Borgia,${ }^{58}$ T. J. V. Bowcock, ${ }^{51}$ E. Bowen, ${ }^{39}$ C. Bozzi, ${ }^{16}$ T. Brambach,,${ }^{9}$ J. van den Brand,${ }^{41}$ J. Bressieux ${ }^{38}$ D. Brett,${ }^{53}$ M. Britsch, ${ }^{10}$ T. Britton, ${ }^{58}$ N. H. Brook, ${ }^{45}$ H. Brown, ${ }^{51}$ A. Bursche, ${ }^{39}$ G. Busetto, ${ }^{21, q}$ J. Buytaert, ${ }^{37}$ S. Cadeddu, ${ }^{15}$ O. Callot, ${ }^{7}$ M. Calvi,${ }^{20, j}$ M. Calvo Gomez, ${ }^{35, n}$ A. Camboni, ${ }^{35}$ P. Campana, ${ }^{18,37}$ D. Campora Perez, ${ }^{37}$ A. Carbone, ${ }^{14, c}$ G. Carboni, ${ }^{23, \mathrm{k}}$ R. Cardinale, ${ }^{19, \mathrm{i}}$ A. Cardini, ${ }^{15}$ H. Carranza-Mejia, ${ }^{49}$ L. Carson, ${ }^{52}$ K. Carvalho Akiba, ${ }^{2}$ G. Casse,${ }^{51}$ L. Cassina, ${ }^{1}$ L. Castillo Garcia, ${ }^{37}$ M. Cattaneo, ${ }^{37}$ Ch. Cauet, ${ }^{9}$ R. Cenci, ${ }^{57}$ M. Charles,${ }^{54} \mathrm{Ph}$. Charpentier, ${ }^{37}$ P. Chen, ${ }^{3,38}$ S.-F. Cheung, ${ }^{54}$ N. Chiapolini, ${ }^{39}$ M. Chrzaszcz, ${ }^{39,25}$ K. Ciba,${ }^{37}$ X. Cid Vidal,${ }^{37}$ G. Ciezarek,${ }^{52}$ P. E. L. Clarke, ${ }^{49}$ M. Clemencic, ${ }^{37}$ H. V. Cliff, ${ }^{46}$ J. Closier,${ }^{37}$ C. Coca,${ }^{28}$ V. Coco, ${ }^{40}$ J. Cogan, ${ }^{6}$ E. Cogneras, ${ }^{5}$ P. Collins, ${ }^{37}$ A. Comerma-Montells, ${ }^{35}$ A. Contu, ${ }^{15,37}$ A. Cook,${ }^{45}$ M. Coombes,${ }^{45}$ S. Coquereau, ${ }^{8}$ G. Corti, ${ }^{37}$ B. Couturier, ${ }^{37}$ G. A. Cowan, ${ }^{49}$ D. C. Craik,${ }^{47}$ S. Cunliffe,,${ }^{52}$ R. Currie,${ }^{49}$ C. D' Ambrosio, ${ }^{37}$ P. David,${ }^{8}$ P. N. Y. David, ${ }^{40}$ A. Davis ${ }^{56}$ I. De Bonis, ${ }^{4}$ K. De Bruyn,${ }^{40}$ S. De Capua,${ }^{53}$ M. De Cian, ${ }^{11}$ J. M. De Miranda, ${ }^{1}$ L. De Paula, ${ }^{2}$ W. De Silva, ${ }^{56}$ P. De Simone, ${ }^{18}$ D. Decamp, ${ }^{4}$ M. Deckenhoff,,${ }^{9}$ L. Del Buono,${ }^{8}$ N. Déléage, ${ }^{4}$ D. Derkach,${ }^{54}$ O. Deschamps,${ }^{5}$ F. Dettori, ${ }^{41}$ A. Di Canto, ${ }^{11}$ H. Dijkstra,${ }^{37}$ M. Dogaru, ${ }^{28}$ S. Donleavy, ${ }^{51}$ F. Dordei, ${ }^{11}$ A. Dosil Suárez,${ }^{36}$ D. Dossett ${ }^{47}$ A. Dovbnya,${ }^{42}$ F. Dupertuis, ${ }^{38}$ P. Durante,${ }^{37}$ R. Dzhelyadin,${ }^{34}$ A. Dziurda ${ }^{25}$ A. Dzyuba, ${ }^{29}$ S. Easo, ${ }^{48}$ U. Egede,${ }^{52}$ V. Egorychev, ${ }^{30}$ S. Eidelman, ${ }^{33}$ D. van Eijk, ${ }^{40}$ S. Eisenhardt, ${ }^{49}$ U. Eitschberger, ${ }^{9}$ R. Ekelhof, ${ }^{9}$ L. Eklund,${ }^{50,37}$ I. El Rifai, ${ }^{5}$ Ch. Elsasser, ${ }^{39}$ A. Falabella, ${ }^{14, \mathrm{e}}$ C. Färber,${ }^{11}$ C. Farinelli,${ }^{40}$ S. Farry,${ }^{51}$ D. Ferguson, ${ }^{49}$

V. Fernandez Albor, ${ }^{36}$ F. Ferreira Rodrigues, ${ }^{1}$ M. Ferro-Luzzi, ${ }^{37}$ S. Filippov,${ }^{32}$ M. Fiore, ${ }^{16, e}$ C. Fitzpatrick,${ }^{37}$ M. Fontana,${ }^{10}$ F. Fontanelli, ${ }^{19, i}$ R. Forty, ${ }^{37}$ O. Francisco, ${ }^{2}$ M. Frank,${ }^{37}$ C. Frei,${ }^{37}$ M. Frosini, ${ }^{17,37, f}$ E. Furfaro, ${ }^{23, k}$ A. Gallas Torreira, ${ }^{36}$ D. Galli, ${ }^{14, \mathrm{c}}$ M. Gandelman, ${ }^{2}$ P. Gandini, ${ }^{58}$ Y. Gao, ${ }^{3}$ J. Garofoli, ${ }^{58}$ P. Garosi, ${ }^{53}$ J. Garra Tico, ${ }^{46}$ L. Garrido, ${ }^{35}$ C. Gaspar, ${ }^{37}$ R. Gauld,${ }^{54}$ E. Gersabeck, ${ }^{11}$ M. Gersabeck,${ }^{53}$ T. Gershon, ${ }^{47}$ Ph. Ghez, ${ }^{4}$ V. Gibson, ${ }^{46}$ L. Giubega,${ }^{28}$ V. V. Gligorov, ${ }^{37}$ C. Göbel,${ }^{59}$ D. Golubkov, ${ }^{30}$ A. Golutvin, ${ }^{52,30,37}$ A. Gomes, ${ }^{2}$ P. Gorbounov, ${ }^{30,37}$ H. Gordon, ${ }^{37}$ M. Grabalosa Gándara, ${ }^{5}$ R. Graciani Diaz, ${ }^{35}$ L. A. Granado Cardoso, ${ }^{37}$ E. Graugés, ${ }^{35}$ G. Graziani, ${ }^{17}$ A. Grecu, ${ }^{28}$ E. Greening ${ }^{54}$ S. Gregson, ${ }^{46}$ P. Griffith, ${ }^{44}$ O. Grünberg,${ }^{60}$ B. Gui,${ }^{58}$ E. Gushchin, ${ }^{32}$ Yu. Guz,${ }^{34,37}$ T. Gys,${ }^{37}$ C. Hadjivasiliou, ${ }^{58}$ G. Haefeli, ${ }^{38}$ C. Haen,${ }^{37}$ S. C. Haines, ${ }^{46}$ S. Hall,${ }^{52}$ B. Hamilton,${ }^{57}$ T. Hampson, ${ }^{45}$ S. Hansmann-Menzemer, ${ }^{11}$ N. Harnew,${ }^{54}$ S. T. Harnew, ${ }^{45}$ J. Harrison, ${ }^{53}$ T. Hartmann, ${ }^{60}$ J. He,${ }^{37}$ T. Head,${ }^{37}$ V. Heijne, ${ }^{40}$ K. Hennessy, ${ }^{51}$ P. Henrard, ${ }^{5}$ J. A. Hernando Morata, ${ }^{36}$ E. van Herwijnen,${ }^{37}$ M. Heß,${ }^{60}$ A. Hicheur, ${ }^{1}$ E. Hicks, ${ }^{51}$ D. Hill, ${ }^{54}$ M. Hoballah, ${ }^{5}$ C. Hombach, ${ }^{53}$ W. Hulsbergen,${ }^{40}$ P. Hunt,${ }^{54}$ T. Huse,${ }^{51}$ N. Hussain, ${ }^{54}$ D. Hutchcroft,,${ }^{51}$ D. Hynds,${ }^{50}$ V. Iakovenko, ${ }^{43}$ M. Idzik,${ }^{26}$ P. Ilten, ${ }^{12}$ R. Jacobsson, ${ }^{37}$ A. Jaeger, ${ }^{11}$ E. Jans, ${ }^{40}$ P. Jaton, ${ }^{38}$ A. Jawahery,${ }^{57}$ F. Jing, ${ }^{3}$ M. John,${ }^{54}$ D. Johnson, ${ }^{54}$ C. R. Jones, ${ }^{46}$ C. Joram,${ }^{37}$ B. Jost, ${ }^{37}$ M. Kaballo, ${ }^{9}$ S. Kandybei, ${ }^{42}$ W. Kanso, ${ }^{6}$ M. Karacson, ${ }^{37}$ T. M. Karbach,${ }^{37}$ I. R. Kenyon, ${ }^{44}$ T. Ketel, ${ }^{41}$ B. Khanji, ${ }^{20}$ O. Kochebina, ${ }^{7}$ I. Komarov, ${ }^{38}$

R. F. Koopman, ${ }^{41}$ P. Koppenburg, ${ }^{40}$ M. Korolev, ${ }^{31}$ A. Kozlinskiy, ${ }^{40}$ L. Kravchuk, ${ }^{32}$ K. Kreplin, ${ }^{11}$ M. Kreps, ${ }^{47}$ G. Krocker, ${ }^{11}$ P. Krokovny, ${ }^{33}$ F. Kruse, ${ }^{9}$ M. Kucharczyk,${ }^{20,25,37, j}$ V. Kudryavtsev, ${ }^{33}$ K. Kurek, ${ }^{27}$ T. Kvaratskheliya,${ }^{30,37}$ V. N. La Thi, ${ }^{38}$ D. Lacarrere, ${ }^{37}$ G. Lafferty,${ }^{53}$ A. Lai, ${ }^{15}$ D. Lambert, ${ }^{49}$ R. W. Lambert, ${ }^{41}$ E. Lanciotti, ${ }^{37}$ G. Lanfranchi, ${ }^{18}$ C. Langenbruch, ${ }^{37}$ T. Latham, ${ }^{47}$ C. Lazzeroni,${ }^{44}$ R. Le Gac, ${ }^{6}$ J. van Leerdam, ${ }^{40}$ J.-P. Lees, ${ }^{4}$ R. Lefèvre, ${ }^{5}$ A. Leflat,${ }^{31}$ J. Lefrançois, ${ }^{7}$ S. Leo, ${ }^{22}$ O. Leroy, ${ }^{6}$ T. Lesiak, ${ }^{25}$ B. Leverington, ${ }^{11}$ Y. Li, ${ }^{3}$ L. Li Gioi, ${ }^{5}$ M. Liles,${ }^{51}$ R. Lindner, ${ }^{37}$ C. Linn, ${ }^{11}$ B. Liu, ${ }^{3}$ G. Liu, ${ }^{37}$ S. Lohn,${ }^{37}$ I. Longstaff, ${ }^{50}$ J. H. Lopes, ${ }^{2}$ N. Lopez-March,${ }^{38}$ H. Lu, ${ }^{3}$ D. Lucchesi, ${ }^{21, q}$ J. Luisier, ${ }^{38}$ H. Luo, ${ }^{49}$ O. Lupton, ${ }^{54}$ F. Machefert, ${ }^{7}$ I. V. Machikhiliyan, ${ }^{4,30}$ F. Maciuc, ${ }^{28}$ O. Maev, ${ }^{29,37}$ S. Malde, ${ }^{54}$ 
G. Manca, ${ }^{15, \mathrm{~d}}$ G. Mancinelli, ${ }^{6}$ J. Maratas, ${ }^{5}$ U. Marconi, ${ }^{14}$ P. Marino, ${ }^{22, \mathrm{~s}}$ R. Märki,${ }^{38}$ J. Marks, ${ }^{11}$ G. Martellotti, ${ }^{24}$ A. Martens, ${ }^{8}$ A. Martín Sánchez, ${ }^{7}$ M. Martinelli, ${ }^{40}$ D. Martinez Santos, ${ }^{41,37}$ D. Martins Tostes, ${ }^{2}$ A. Martynov,${ }^{31}$ A. Massafferri, ${ }^{1}$ R. Matev, ${ }^{37}$ Z. Mathe,${ }^{37}$ C. Matteuzzi,${ }^{20}$ E. Maurice, ${ }^{6}$ A. Mazurov,${ }^{16,32,37, e}$ J. McCarthy, ${ }^{44}$ A. McNab, ${ }^{53}$ R. McNulty, ${ }^{12}$ B. McSkelly,${ }^{51}$ B. Meadows,${ }^{56,54}$ F. Meier, ${ }^{9}$ M. Meissner, ${ }^{11}$ M. Merk, ${ }^{40}$ D. A. Milanes, ${ }^{8}$ M.-N. Minard, ${ }^{4}$ J. Molina Rodriguez, ${ }^{59}$ S. Monteil, ${ }^{5}$ D. Moran,${ }^{53}$ P. Morawski, ${ }^{25}$ A. Mordà, ${ }^{6}$ M. J. Morello, ${ }^{22, s}$ R. Mountain, ${ }^{58}$ I. Mous, ${ }^{40}$ F. Muheim, ${ }^{49}$ K. Müller,${ }^{39}$ R. Muresan, ${ }^{28}$ B. Muryn,${ }^{26}$ B. Muster,${ }^{38}$ P. Naik, ${ }^{45}$ T. Nakada, ${ }^{38}$ R. Nandakumar, ${ }^{48}$ I. Nasteva, ${ }^{1}$ M. Needham, ${ }^{49}$ S. Neubert,${ }^{37}$ N. Neufeld, ${ }^{37}$ A. D. Nguyen, ${ }^{38}$ T. D. Nguyen, ${ }^{38}$ C. Nguyen-Mau, ${ }^{38, o}$ M. Nicol, ${ }^{7}$ V. Niess, ${ }^{5}$ R. Niet, ${ }^{9}$ N. Nikitin, ${ }^{31}$ T. Nikodem,,${ }^{11}$ A. Nomerotski, ${ }^{54}$ A. Novoselov, ${ }^{34}$ A. Oblakowska-Mucha, ${ }^{26}$ V. Obraztsov, ${ }^{34} \mathrm{~S}$. Oggero, ${ }^{40} \mathrm{~S}$. Ogilvy,${ }^{50} \mathrm{O}$. Okhrimenko, ${ }^{43} \mathrm{R}$. Oldeman, ${ }^{15, \mathrm{~d}}$

M. Orlandea, ${ }^{28}$ J. M. Otalora Goicochea,${ }^{2}$ P. Owen, ${ }^{52}$ A. Oyanguren, ${ }^{35}$ B. K. Pal,${ }^{58}$ A. Palano, ${ }^{13, b}$ M. Palutan, ${ }^{18}$

J. Panman ${ }^{37}$ A. Papanestis,${ }^{48}$ M. Pappagallo, ${ }^{50}$ C. Parkes,${ }^{53}$ C. J. Parkinson,${ }^{52}$ G. Passaleva, ${ }^{17}$ G. D. Patel,${ }^{51}$ M. Patel,${ }^{52}$ G. N. Patrick, ${ }^{48}$ C. Patrignani, ${ }^{19, \mathrm{i}}$ C. Pavel-Nicorescu, ${ }^{28}$ A. Pazos Alvarez,${ }^{36}$ A. Pearce, ${ }^{53}$ A. Pellegrino, ${ }^{40}$ G. Penso, ${ }^{24,1}$ M. Pepe Altarelli, ${ }^{37}$ S. Perazzini, ${ }^{14, c}$ E. Perez Trigo, ${ }^{36}$ A. Pérez-Calero Yzquierdo,${ }^{35}$ P. Perret, ${ }^{5}$ M. Perrin-Terrin, ${ }^{6}$ L. Pescatore, ${ }^{44}$ E. Pesen, ${ }^{61}$ G. Pessina,${ }^{20}$ K. Petridis,${ }^{52}$ A. Petrolini, ${ }^{19, \mathrm{i}}$ A. Phan, ${ }^{58}$ E. Picatoste Olloqui, ${ }^{35}$ B. Pietrzyk,${ }^{4}$ T. Pilar, ${ }^{47}$ D. Pinci,${ }^{24}$ S. Playfer,${ }^{49}$ M. Plo Casasus, ${ }^{36}$ F. Polci, ${ }^{8}$ G. Polok,${ }^{25}$ A. Poluektov, ${ }^{47,33}$ E. Polycarpo,${ }^{2}$ A. Popov,${ }^{34}$ D. Popov, ${ }^{10}$ B. Popovici, ${ }^{28}$ C. Potterat,${ }^{35}$ A. Powell, ${ }^{54}$ J. Prisciandaro, ${ }^{38}$ A. Pritchard ${ }^{51}$ C. Prouve, ${ }^{7}$ V. Pugatch, ${ }^{43}$ A. Puig Navarro, ${ }^{38}$ G. Punzi, ${ }^{22, r}$ W. Qian, ${ }^{4}$ J. H. Rademacker, ${ }^{45}$ B. Rakotomiaramanana, ${ }^{38}$ M. S. Rangel, ${ }^{2}$ I. Raniuk,${ }^{42}$ N. Rauschmayr, ${ }^{37}$ G. Raven, ${ }^{41}$ S. Redford ${ }^{54}$ M. M. Reid ${ }^{47}$ A. C. dos Reis, ${ }^{1}$ S. Ricciardi, ${ }^{48}$ A. Richards,${ }^{52}$ K. Rinnert, ${ }^{51}$ V. Rives Molina, ${ }^{35}$ D. A. Roa Romero, ${ }^{5}$ P. Robbe, ${ }^{7}$ D. A. Roberts, ${ }^{57}$ A. B. Rodrigues, ${ }^{1}$ E. Rodrigues, ${ }^{53}$ P. Rodriguez Perez,${ }^{36}$ S. Roiser, ${ }^{37}$ V. Romanovsky,${ }^{34}$ A. Romero Vidal, ${ }^{36}$ J. Rouvinet, ${ }^{38}$ T. Ruf,${ }^{37}$ F. Ruffini, ${ }^{22}$ H. Ruiz, ${ }^{35}$ P. Ruiz Valls, ${ }^{35}$ G. Sabatino, ${ }^{24, k}$ J. J. Saborido Silva, ${ }^{36}$ N. Sagidova, ${ }^{29}$ P. Sail,${ }^{50}$ B. Saitta, ${ }^{15, d}$ V. Salustino Guimaraes, ${ }^{2}$ B. Sanmartin Sedes,${ }^{36}$ R. Santacesaria ${ }^{24}$ C. Santamarina Rios,${ }^{36}$ E. Santovetti, ${ }^{23, \mathrm{k}}$ M. Sapunov, ${ }^{6}$ A. Sarti, ${ }^{18}$ C. Satriano, ${ }^{24, \mathrm{~m}}$ A. Satta ${ }^{23}$ M. Savrie, ${ }^{16, e}$ D. Savrina, ${ }^{30,31}$ M. Schiller, ${ }^{41}$ H. Schindler, ${ }^{37}$ M. Schlupp, ${ }^{9}$ M. Schmelling, ${ }^{10}$ B. Schmidt, ${ }^{37}$ O. Schneider, ${ }^{38}$ A. Schopper, ${ }^{37}$ M.-H. Schune, ${ }^{7}$ R. Schwemmer, ${ }^{37}$ B. Sciascia, ${ }^{18}$ A. Sciubba, ${ }^{24}$ M. Seco, ${ }^{36}$ A. Semennikov, ${ }^{30}$ K. Senderowska, ${ }^{26}$ I. Sepp ${ }^{52}$ N. Serra, ${ }^{39}$ J. Serrano, ${ }^{6}$ P. Seyfert, ${ }^{11}$ M. Shapkin, ${ }^{34}$ I. Shapoval,,${ }^{16,42, e}$ P. Shatalov, ${ }^{30}$ Y. Shcheglov, ${ }^{29}$ T. Shears, ${ }^{51}$ L. Shekhtman, ${ }^{33}$ O. Shevchenko, ${ }^{42}$ V. Shevchenko, ${ }^{30}$ A. Shires, ${ }^{9}$ R. Silva Coutinho, ${ }^{47}$ M. Sirendi, ${ }^{46}$ N. Skidmore, ${ }^{45}$ T. Skwarnicki, ${ }^{58}$ N. A. Smith,${ }^{51}$ E. Smith,${ }^{54,48}$ E. Smith, ${ }^{52}$ J. Smith, ${ }^{46}$ M. Smith, ${ }^{53}$ M. D. Sokoloff, ${ }^{56}$ F. J. P. Soler, ${ }^{50}$ F. Soomro, ${ }^{38}$ D. Souza, ${ }^{45}$ B. Souza De Paula, ${ }^{2}$ B. Spaan, ${ }^{9}$ A. Sparkes, ${ }^{49}$ P. Spradlin,${ }^{50}$ F. Stagni,${ }^{37}$ S. Stahl,${ }^{11}$ O. Steinkamp, ${ }^{39}$ S. Stevenson, ${ }^{54}$ S. Stoica, ${ }^{28}$ S. Stone,${ }^{58}$ B. Storaci, ${ }^{39}$ M. Straticiuc, ${ }^{28}$ U. Straumann, ${ }^{39}$ V. K. Subbiah, ${ }^{37}$ L. Sun,${ }^{56}$ W. Sutcliffe,${ }^{52}$ S. Swientek,,${ }^{9}$ V. Syropoulos,${ }^{41}$ M. Szczekowski, ${ }^{27}$ P. Szczypka, ${ }^{38,37}$ D. Szilard, ${ }^{2}$ T. Szumlak, ${ }^{26}$ S. T' Jampens, ${ }^{4}$ M. Teklishyn, ${ }^{7}$ E. Teodorescu, ${ }^{28}$

F. Teubert,${ }^{37}$ C. Thomas,${ }^{54}$ E. Thomas, ${ }^{37}$ J. van Tilburg, ${ }^{11}$ V. Tisserand, ${ }^{4}$ M. Tobin,${ }^{38}$ S. Tolk, ${ }^{41}$ D. Tonelli, ${ }^{37}$ S. Topp-Joergensen, ${ }^{54}$ N. Torr,${ }^{54}$ E. Tournefier, ${ }^{4,52}$ S. Tourneur, ${ }^{38}$ M. T. Tran, ${ }^{38}$ M. Tresch, ${ }^{39}$ A. Tsaregorodtsev, ${ }^{6}$

P. Tsopelas,${ }^{40}$ N. Tuning, ${ }^{40,37}$ M. Ubeda Garcia ${ }^{37}$ A. Ukleja, ${ }^{27}$ A. Ustyuzhanin, ${ }^{52, p}$ U. Uwer, ${ }^{11}$ V. Vagnoni, ${ }^{14}$

G. Valenti, ${ }^{14}$ A. Vallier, ${ }^{7}$ R. Vazquez Gomez, ${ }^{18}$ P. Vazquez Regueiro, ${ }^{36}$ C. Vázquez Sierra, ${ }^{36}$ S. Vecchi, ${ }^{16}$

J. J. Velthuis, ${ }^{45}$ M. Veltri, ${ }^{17, \mathrm{~g}}$ G. Veneziano, ${ }^{38}$ M. Vesterinen, ${ }^{37}$ B. Viaud, ${ }^{7}$ D. Vieira, ${ }^{2}$ X. Vilasis-Cardona, ${ }^{35, n}$ A. Vollhardt, ${ }^{39}$ D. Volyanskyy, ${ }^{10}$ D. Voong, ${ }^{45}$ A. Vorobyev, ${ }^{29}$ V. Vorobyev, ${ }^{33}$ C. Voß,${ }^{60}$ H. Voss, ${ }^{10}$ J. A. de Vries, ${ }^{40}$ R. Waldi, ${ }^{60}$ C. Wallace, ${ }^{47}$ R. Wallace, ${ }^{12}$ S. Wandernoth, ${ }^{11}$ J. Wang,${ }^{58}$ D. R. Ward, ${ }^{46}$ N. K. Watson,,${ }^{44}$ A. D. Webber, ${ }^{53}$ D. Websdale, ${ }^{52}$ M. Whitehead, ${ }^{47}$ J. Wicht,${ }^{37}$ J. Wiechczynski, ${ }^{25}$ D. Wiedner, ${ }^{11}$ L. Wiggers, ${ }^{40}$ G. Wilkinson, ${ }^{54}$ M. P. Williams, ${ }^{47,48}$ M. Williams, ${ }^{55}$ F. F. Wilson, ${ }^{48}$ J. Wimberley, ${ }^{57}$ J. Wishahi, ${ }^{9}$ W. Wislicki, ${ }^{27}$ M. Witek, ${ }^{25}$ S. A. Wotton, ${ }^{46}$ S. Wright,${ }^{46}$ S. Wu, ${ }^{3}$ K. Wyllie, ${ }^{37}$ Y. Xie,${ }^{49,37}$ Z. Xing, ${ }^{58}$ Z. Yang, ${ }^{3}$ X. Yuan,${ }^{3}$ O. Yushchenko, ${ }^{34}$ M. Zangoli, ${ }^{14}$ M. Zavertyaev, ${ }^{10, a}$ F. Zhang, ${ }^{3}$ L. Zhang,${ }^{58}$ W. C. Zhang, ${ }^{12}$ Y. Zhang, ${ }^{3}$ A. Zhelezov, ${ }^{11}$ A. Zhokhov, ${ }^{30}$ L. Zhong, ${ }^{3}$ and A. Zvyagin ${ }^{37}$

\title{
(LHCb Collaboration)
}

\author{
${ }^{1}$ Centro Brasileiro de Pesquisas Físicas (CBPF), Rio de Janeiro, Brazil \\ ${ }^{2}$ Universidade Federal do Rio de Janeiro (UFRJ), Rio de Janeiro, Brazil \\ ${ }^{3}$ Center for High Energy Physics, Tsinghua University, Beijing, China \\ ${ }^{4} L A P P$, Université de Savoie, CNRS/IN2P3, Annecy-Le-Vieux, France \\ ${ }^{5}$ Clermont Université, Université Blaise Pascal, CNRS/IN2P3, LPC, Clermont-Ferrand, France
}




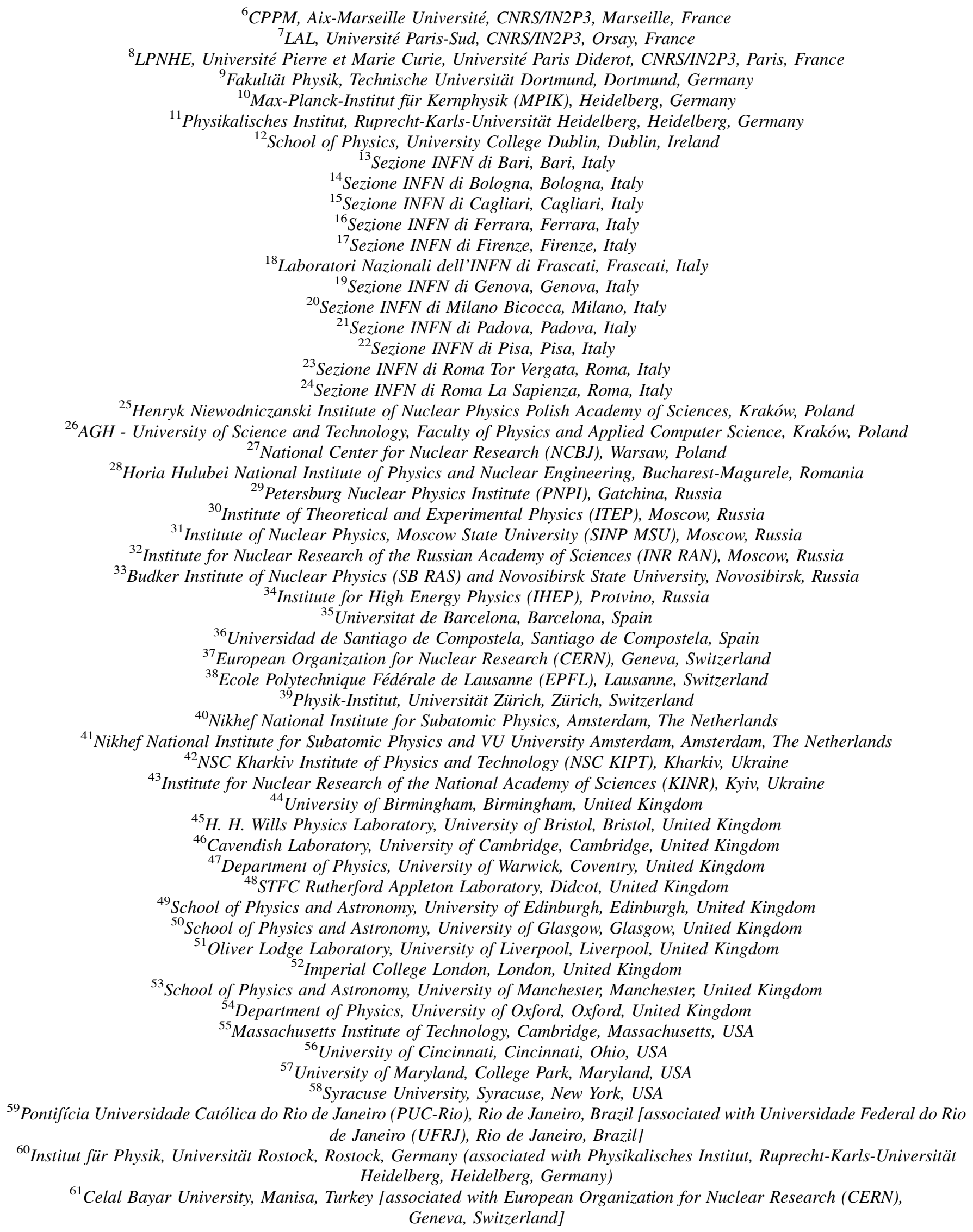


${ }^{a}$ Also at P.N. Lebedev Physical Institute, Russian Academy of Science (LPI RAS), Moscow, Russia.

${ }^{\mathrm{b}}$ Also at Università di Bari, Bari, Italy.

${ }^{\mathrm{c}}$ Also at Università di Bologna, Bologna, Italy.

${ }^{\mathrm{d}}$ Also at Università di Cagliari, Cagliari, Italy.

eAlso at Università di Ferrara, Ferrara, Italy.

${ }^{\mathrm{f}}$ Also at Università di Firenze, Firenze, Italy.

${ }^{\mathrm{g}}$ Also at Università di Urbino, Urbino, Italy.

${ }^{\mathrm{h}}$ Also at Università di Modena e Reggio Emilia, Modena, Italy.

${ }^{\mathrm{i}}$ Also at Università di Genova, Genova, Italy.

${ }^{\mathrm{j}}$ Also at Università di Milano Bicocca, Milano, Italy.

${ }^{\mathrm{k}}$ Also at Università di Roma Tor Vergata, Roma, Italy.

${ }^{1}$ Also at Università di Roma La Sapienza, Roma, Italy.

${ }^{\mathrm{m}}$ Also at Università della Basilicata, Potenza, Italy.

${ }^{\mathrm{n}}$ Also at LIFAELS, La Salle, Universitat Ramon Llull, Barcelona, Spain.

${ }^{\circ}$ Also at Hanoi University of Science, Hanoi, Vietnam.

${ }^{\mathrm{p}}$ Also at Institute of Physics and Technology, Moscow, Russia.

${ }^{\mathrm{q}}$ Also at Università di Padova, Padova, Italy.

${ }^{\mathrm{r}}$ Also at Università di Pisa, Pisa, Italy.

${ }^{\text {s} A l s o ~ a t ~ S c u o l a ~ N o r m a l e ~ S u p e r i o r e, ~ P i s a, ~ I t a l y . ~}$ 